\section{What do we know about the genetics of depression?}

\section{N Martin}

Queensland Institute of Medical Research, Brisbane, Australia

In 2003, Caspi et al. published a landmark paper in Science reporting an interaction, between a polymorphism (5HTTLPR) in the promoter region of the serotonin transporter gene (5HTT renamed SLC6A4) and the number of life events experienced, in predicting risk of depression. This paper has been widely cited and has become the dominant research paradigm in psychiatric genetics because it appears to reconcile the competing claims of genetic and environmental etiology. There have been numerous replication studies, with highly inconsistent results, some supporting the interaction as reported by Caspi, some finding no interaction and others reporting the interaction in the opposite direction. Further, it has recently become apparent that genotyping of the 5HTTLPR polymorphism is somewhat more complicated than previously thought, casting doubts on the veracity of earlier studies. Most importantly, it has been suggested that the GxE detected may be an artifact of the unsatisfactory psychometric properties of the psychiatric constructs being used. We are analyzing data from our large twin family collection (5200 individuals) for whom depression diagnoses, risk factors and DNA samples are available, to bring considerable power to the question of GxE interactions for depression. Further, we are investigating whether the inconsistently replicated interaction of 5HTTLPR (and, independently, any single nucleotide polymorphism haplotype) with stressful life events on risk of depression is moderated by level of social support and whether this could explain the inconsistency. We are also exploring the extent to which IRT modeling will help resolve the effects of GxE from artifacts of measurement.

\section{New insights from cross-linked data on the relationship between psychotic illness and intellectual disability}

\section{VA Morgan}

Neuropsychiatric Epidemiology Research Unit, School of Psychiatry and Clinical Neurosciences, The University of Western Australia, Perth, Australia

Increasingly, national and state health and other registers are being made available for research use. Record linkage across these registers is a cost-effective and efficient means of acquiring large samples and overcoming some of methodological issues facing researchers studying rare outcomes. This presentation describes the methods and benefits of record linkage in the Australian context. To illustrate the points made, it focuses on two studies using cross-linked data from Western Australian population-based registers that are examining the association between schizophrenia and intellectual disability. Although these are rare disorders, both with a lifetime population prevalence of $\sim 1 \%$, the prevalence of schizophrenia among persons with an intellectual disability is reportedly three times the population estimate. However, little is known about the relationship between the two disorders. In the first study, cross-linked data from the intellectual disability register and the psychiatric case register are used to get estimates of the prevalence of comorbidity and to describe the profile of persons with a dual diagnosis of intellectual disability and psychiatric illness, particularly the psychoses. In the second, data from linkage across multiple registers including psychiatric, midwives and intellectual disability registers are used to compare the risk of intellectual disability in children of mothers with schizophrenia and affective psychoses with children of mothers with no recorded psychiatric history. The data are suggestive of underlying genetic risk factors of varying diagnostic specificity. These findings are presented and their etiological implications discussed. The presentation concludes that the facility to integrate records across multiple population databases, sometimes held in separate administrative jurisdictions, has had a marked impact on our capacity to estimate the extent of comorbidity and its prevalence across diagnostic categories, to understand its clinical manifestations and to start to untangle etiological underpinnings.

\section{Dynamin's role in synaptic transmission: a potential target for new antiepileptic drugs}

\section{PJ Robinson \\ Children's Medical Research Institute, Sydney, Australia}

Exocytosis of small synaptic vesicles (SVs) is at the heart of how neurons communicate, by the release of neurotransmitters onto neighboring neurons or muscles. Problems with exocytosis underlie many human diseases, such as schizophrenia or epilepsy. To enable the process to respond rapidly and in a sustainable manner, synaptic vesicle endocytosis (SVE) must retrieve the used, empty SV and recycle it rapidly, to sustain high synaptic transmission rates for more than $1 \mathrm{~min}$. We have been defining the central role of dynamin in the molecular mechanisms of SVE at the synapse. Dynamin is a protein that has multiple 